\title{
SUR TROIS CAS DE CYSTICERCOSE GRAVE CHEZ DES CAMPAGNOLS (RODENTIA-MICROTIDAE)
}

\author{
Par J. BERNARD
}

Les Rongeurs champêtres, tant Muridæ que Microtidæ, peuvent être atteints de diverses affections helminthologiques plus ou moins graves. Entre autres, on trouve assez couramment, dans certaines régions de leur organisme, des larves de Cestodes dont les adultes sont parasites de l'intestin des Carnivores. Les quatre espèces principales que l'on rencontre ainsi en Belgique appartiennent au genre Taenia : ce sont : T. iaeniaeformis Batsch, 1786, T. crassiceps Radolphi, 1810, T. tenuicollis Rudolphi, 1809 et $T$. polyacantha Leuckart, 1856. Les noms de leurs larves sont respectivement: ¿̇ysticercus fasciolaris Rudolphi, 1808, C. longicollis Rudolphi, 1819 . C. talpae Rudolphi, 1819 (= C. innominatus-hypodaei Leuckart, 1857 ) et C. taeniæ-polyacanthre Leuckart, 1856.

Les deux espèces les plus importantes, au point de vue de leur fréquence dans les populations de Rongeurs, sont $T$. taeniaeformis et $T$. tenuicollis. Par contre, au point de vue des répercussions de la présence du parasite sur la santé de l'hôte, celles qui entrainent les conséquences les plus graves sont: T. taeniaeformis et T. crassiceps. Cette note a trait à ces deux dernières espèces.

\section{Taenia taeniaeformis Batsch.}

Ce Cestode, qui à l'état adulte est parasite des Felidæ et de quelques autres Carnassiers, a une larve du type plérocercoïde à scolex non invaginé. A la suite de celui-ci, on trouve une zone rubannée plus ou moins longue. Il s'agit d'une série de segments indifférenciés, résultat de l'activité de la zone génératrice. Cette partie somatique se termine par une petite vessie, dans laquelle débouchent les canaux excréteurs. La longueur de ce pseudostrobile varie de quelques millimètres à plusieurs dizaines de centimètres suivant l'âge de la larve. Celle-ci se trouve presque toujours dans le foie de l'hôte secondaire, enroulée dans une vésicule kystique de la taille d'un pois. 
Au fur et à mesure de la croissance de la partie rubannée, le kyste grossit et entraîne des troubles de plus en plus graves pour l'hôte. Dans la nature, on trouve assez fréquemment des petits Rongeurs dont le poids ne dépasse pas $20 \mathrm{~g}$. et dont le foie contient deux à trois de ces kystes, ce nombre étant même quelquefois dépassé.

Les hôtes secondaires les plus courants de ce Cestode sont les Microtidæ: Microtus arvalis Pall., Arvicola terrestris sherman Shaw., A. t. terrestris L., Pitymys subterraneus de S. L. et Ondatra zibethica L.. On en trouve aussi, mais plus rarement, chez .Microtus agrestis L., Clethrionomys glareolus Schr. et chez les Muridæ.

Au cours de l'année 1957, dans notre élevage, à Gembloux, sont morts deux campagnols aquatiques, Arvicola terrestris terrestris, dont le foie était littéralement envahi de larves de $T$. taeniaeformis. Le premier de ces rongeurs est mort au mois d'août. Cet animal avait été capturé dans le nord du pays, au mois de mars. Etant donné le repos sexuel hivernal, on peut situer sa naissance à l'été 1956 ; son âge était d'environ un an.

A sa mort, cet animal pesait 117,75 g., poids un peu en-dessous de la moyenne pour cette sous-espèce. L'autopsie a révélé que le foie portait 42 gros kystes, contenant chacun une très longue larve de $T$. taeniaeformis (voir photo $\mathrm{n}^{\circ} 1$ ). En réalité, le foie avait complètement disparu; il ne subsistait que quelques isthmes de tissu noble séparant les kystes. Du fait de la présence de cette formidable masse étrangère, tous les autres organes de la cavité abdominale étaient comprimés contre la paroi postérieure. De même, la masse de larves appuyait fortement sur le diaphragme.

En décembre 1957 est mort un autre campagnol de même provenance et d'âge un peu plus avancé, pesant $164 \mathrm{~g}$. à cette date. Son foie contenait 60 kystes de Cysticercus fasciolaris. Leur poids total était de $41,8 \mathrm{~g}$.. En dehors de ces larves, situées dans la cavité péritonéale, le rongeur en portait deux autres, fixées au poumon. Il s'agit là d'une localisation absolument inaccoutumée, pour ne pas dire unique, de strobilocerques de T. taeniaeformis.

\section{Taenia crassiceps Rudolphi.}

Ce Cestode, dont l'adulte parasite l'intestin du renard, Vulpes vulpes L., a une larve qui présente un développement néoténique. Le cysticerque original est enfermé dans une vésicule dont la zone distale se différencie et où se forme une vésicule fille; le développement est ici exogène, contrairement à celui du cénure de Taenia multiceps Leske, 1780. Quelquefois, plusieurs vésicules filles se forment simultanément. Ces dernières restent attachées pendant un 
certain temps à la vésicule mère. Ensuite, elles se détachent et engendrent à leur tour de nouveaux cysticerques parthénogénétiques.

Pendant la période de multiplication chez l'hôte secondaire, certaines larves dévaginent leur scolex et l'on voit apparaitre un début

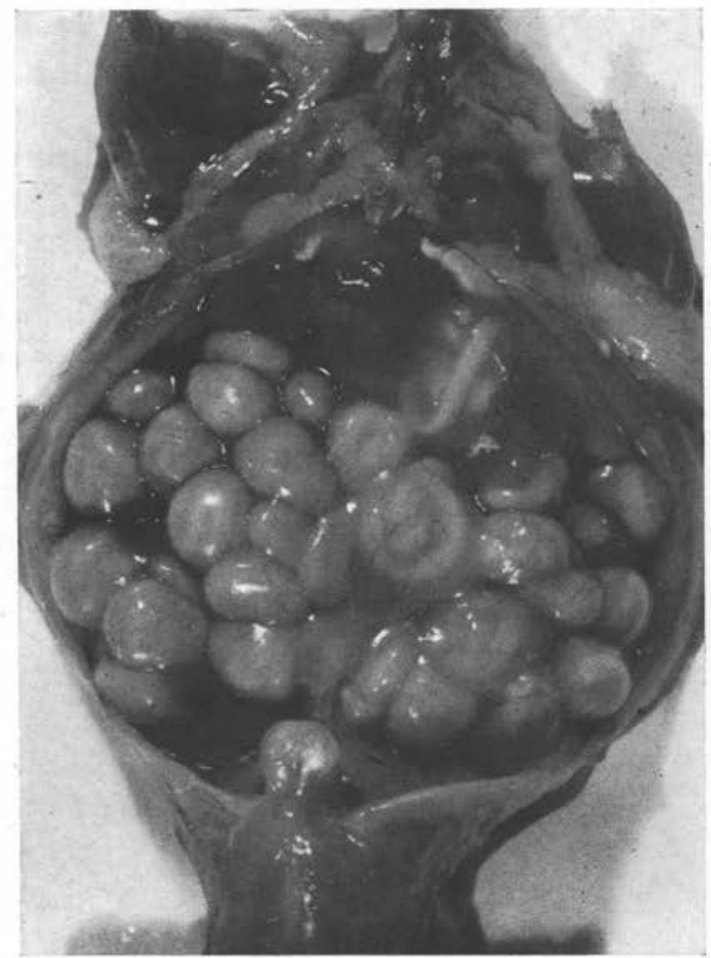

FIG. 1. - Cavité abdominale d'un grand campagnol Arvicola terrestris $\mathrm{L}$. bourrée de larves de Tania taniaformis Batsch.

de segmentation dans la zone musculeuse qui lui fait suite. Toutefois, la longueur de ce strobile larvaire ne dépasse jamais quelques segments, comme chez Taenia polyacantha.

Les Rongeurs capturés dans la nature et qui sont atteints de cette cysticercose portent de quelques dizaines à quelques centaines de cysticerques. En général, ceux-ci sont localisés sous la peau du cou, des aisselles ou de l'aine; on en trouve rarement dans la cavité générale proprement dite. 
En juin 1953 est morte dans notre élevage de campagnols des champs, Microtus arvalis Pall., une femelle qui avait été capturée adulte en mars de la même année. La date de la capture permet de supposer qu'il s'agit d'un animal né en août 1952 et qui avait donc environ dix mois lors de sa mort.

Lors de son introduction dans l'élevage, cet animal pesait 27,10 g.. Accouplée avec un mâle, le 18 avril, elle donnait naissance à trois jeunes le 14 mai. A la date de sa mort, le $1^{\text {er }}$ juin 1953, elle pesait 25,05 g.. L'autopsie a révélé la présence d'une multitude de cysticerques de Taenia crassiceps, de quatre strobilocerques de $T$. taeniaeformis dans le foie et d'un cinquième calcifié et tombé dans la cavité viscérale, où il fut trouvé reposant sur les intestins.

Le phénomène le plus caractéristique était indiscutablement la présence des Cysticercus taeniz-crassicipitis. La cavité générale était complètement envahie de larves de ce cestode, depuis la région thyroïdienne jusqu'à l'anus. La grande majorité des vésicules étaient libres, mais certaines d'entre elles étaient fixées, comme greffées, sur des organes tels que les poumons, le foie et la rate. Le dénombrement exact des cysticerques était difficile. Le comptage que nous avons effectué nous a donné entre trois mille quatre cents et trois mille cinq cents parasites; leur volume total était d'environ huit centimètres cubes. Ce nombre extraordinairement élevé de cysticerques, joint aux quatre strobilocerques du foie, est amplement suffisant pour expliquer la mort du rongeur.

L'examen attentif des larves de $T$. crassiceps a montré que la plupart d'entre elles étaient ornées de nombreuses vésicules filles, à des degrés divers de développement. Le fait est d'autant plus curieux que les larves de cette espèce, que l'on trouve dans des rongeurs capturés dans la nature, sont en général dépourvues de ces vésicules filles. Tout au plus observe-t-on des «bouquets » de cysticerques, reliés par leurs régions distales. Rausch (2) affirme même qu'il n'a jamais rencontré de larve de cette espèce qui montrait des signes de paedogénèse nette. Or, ici, pratiquement tous les cysticerques rencontrés montraient des signes de multiplication intense, comme si le processus s'était accéléré au fur et à mesure de l'augmentation du nombre de descendants néoténiques et que le phénomène ait présenté un caractère de « malignité ». Il est évidemment trop tôt pour faire la moindre supposition à cet égard ou pour chercher si la cause de ce phénomène est d'ordre interne ou externe, mais le fait méritait d'être signalé.

Arvy (1) est le seul auteur, à notre connaissance, qui ait décrit un cas de cysticercose intense due à Cysticercus taeniæ-crassicipitis chez le campagnol des champs. Cet auteur, qui a donné une description précise et complète des lésions observées chez l'hôte, a mis en rapport la présence de ces parasites avec un arrêt de l'activité testiculaire. Le tractus génital du mâle, objet de ses travaux, avait l'aspect de celui d'un animal dont les canaux déférents auraient 
été ligaturés. Il s'agit sans doute là d'une simple compression mécanique accidentelle, due à la présence de nombreux cysticerques dans la cavité pelvienne. La naissance de trois jeunes, quinze jours avant la mort de la femelle du campagnol, objet de nos observations, montre que ce phénomène de stérilisation de l'hôte n'est pas un cas général.

\section{Discussion}

Bien que les trois cas d'hyperparasitose que nous venons de décrire présentent des points communs, il nous paraît difficile de les discuter simultanément. En effet, la genèse de cette abondance de parasites chez un seul hôte est essentiellement différente dans le cas de Taenia taeniaeformis et dans celui de T. crassiceps. Chez le premier de ces Cestodes, toute pædogénèse paraît exclue et, par conséquent, l'abondance des strobilocerques ne peut être due qu'à de la surinfestation. Au contraire, les larves du second sont bien connues comme étant susceptibles de multiplication parthénogénetique. Nous envisagerons donc ici aussi les deux espèces séparément.

\section{a) Taenia taeniaeformis.}

Comme nous le signalons dans l'introduction, on trouve quelquefois des petits campagnols porteurs de trois et même de quatre kystes de Cysticercus fasciolaris, bien que ces chiffres soient assez rares. Dans la nature, il semble que les grands campagnols, Arvicola terrestris subsp., soient moins atteints que leur cousin. En effet, sur les quarante-deux animaux de cette espèce que nous avons trouvés porteurs de cysticerques de ce Taenia, trente-quatre contenaient un kyste, sept en recélaient deux et un seul Rongeur avait le foie envahi par quatre de ces larves. Dans ce dernier cas, il s'agissait d'un animal ayant vécu quelques mois en élevage avant de mourir.

Il est done quasiment certain que les deux cas de campagnols dont le foie était envahi de plusieurs dizaines de strobilocerques sont exceptionnels et dus aux conditions de l'élevage. Les causes ayant entrainé cette hyperparasitose peuvent être de deux natures : $1^{\circ}$ les conditions artificielles ont favorisé la survie de l'animal malgré la gravité de son état; $2^{\circ}$ au contraire, ces conditions ont entraìné des infestations secondaires anormales.

Nous ne saurions discuter ici de l'importance relative des divers facteurs de mortalité dans la nature. Toutefois, il est un fait d'ob- 
servation indiscutable, c'est qu'au sein des populations naturelles de Mammifères, la moindre perte de vitalité d'un individu lui est rapidement fatale, étant donné la pression biotique à laquelle il est soumis (action de prédateurs, compétition intraspécifique, etc...). La présence de quelques larves de $T$. taeniaeformis seulement est sans doute suffisante pour entraîner rapidement la disparition de l'individu affecté. De ce fait, aucun animal n'aurait atteint le degré de cachexie provoqué par un aussi grand nombre de kystes dans le foie.

Cependant, on pourrait supposer que les chances de contamination dans la nature sont telles qu'un Rongeur puisse absorber plusieurs dizaines d'embryons hexacanthes de T. taeniaeformis. Dans cette hypothèse, nous aurions introduit dans l'élevage deux Arvicola dont l'organisme recélait déjà, plus ou moins développées, une cinquantaine de larves de $T$. taeniaeformis. A l'abri du \& struggle for life », ils auraient survécu malgré la gravité de leur état. Nous ne pensons toutefois pas que cette explication puisse s'appliquer à notre observation. En effet, si les chances de contamination étaient aussi grandes dans la nature, on devrait au moins trouver quelquefois des Rongeurs portant plus d'un ou deux strobilocerques plus ou moins développés. L'écart entre les chiffres observés chez des animaux provenant de populations naturelles et chez les deux campagnols en question nous parait trop grand pour admettre que ces derniers portaient déjà leurs dizaines de larves au moment de leur introduction au laboratoire.

Dans ce cas, il faut admettre une surinfestation anormale due aux conditions de l'élevage. Celle-ci est en effet très plausible. Ces animaux furent nourris de betteraves et d'avoine aplatie. Or, celleci provient d'une ferme voisine fréquentée par de nombreux chats. Il est certain que cet aliment constitue une source importante d'œufs de T. taeniaformis, amenés là avec les excréments de ce Félidé domestique. Une preuve de la surinfestation est d'ailleurs fournie par la présence de deux strobilocerques sur le poumon de l'un des deux Arvicola.

Cette localisation inaccoutumée est difficilement explicable.

On peut concevoir que l'invasion du foie par les larves de T. taeniaeformis puisse se faire par deux voies. D'une part, les embryons hexacanthes, une fois dans l'estomac, peuvent remonter le cholédoque et envahir le tissu hépatique. D'autre part, et c'est le cas le plus courant chez les larves de Cestode, de l'estomac, ils peuvent pénétrer dans le courant sanguin et se laisser charrier passivement jusqu'au moment où ils arrivent dans un organe ou un tissu qui leur convienne. 
Si la voie normale de pénétration est le cholédoque, on peut admettre que la masse de strobilocerques déjà présente ait obturé par simple pression mécanique ce canal et ainsi fermé le chemin d'accès aux derniers venus. Ceux-ci auraient alors, soit activement, soit passivement à la faveur d'une hémorragie, pénétré dans le courant sanguin et se seraient par la suite fixés au poumon. $\mathrm{Si}$, au contraire, l'invasion se fait par le système circulatoire, il est difficile de concevoir que ses voies d'accès aient été obturées par les premières larves.

On admet généralement que les embryons qui sont charriés passivement par le sang dégénèrent lorsqu'ils arrivent dans un organe qui ne leur convient pas. Le fait est prouvé en ce qui concerne les larves de Taenia multiceps. Lorsque celles-ci n'atteignent pas le tissu nerveux du mouton, par exemple, elles meurent et sont phagocytées. Il est possible que cette inadaptation dans certains organes soit due à une réaction active de l'hôte dans ces zones. Dans ce cas, on peut admettre que, débilité par les premiers cysticerques fixés dans le foie, le campagnol ait offert une résistance moindre à de nouvelles contaminations et que des tissus, normalement résistants à l'invasion par les embryons de T. taeniaformis, soient devenus colonisables par ceux-ci.

\section{b) Taenia crassiceps.}

Dans ce cas, également, l'hypercysticercose observée est due aux conditions artificielles du milieu. En effet, comme nous l'avons signalé, les Rongeurs atteints de Cysticercus taeniæ-crassicipitis que l'on trouve dans la nature ne portent jamais que quelques centaines de cysticerques au maximum, localisés aux tissus souscutanés. Notons à ce propos que les animaux examinés par Arvy (1) avaient également passé six mois en élevage avant d'être autopsiés.

Les causes artificielles ayant entrainé l'aggravation de l'affection pourraient être de même nature que dans le cas précédent. Or, ici, la surinfestation est absolument à écarter. En effet, le lieu d'élevage et l'endroit d'origine des aliments ne sont pas fréquentés par les renards. Jamais d'ailleurs, cette cysticercose n'a été observée chez des animaux nés au laboratoire. Donc, ce sont bien les conditions d'élevage qui ont favorisé la survie du Rongeur malgré la gravité de l'affection dont il était atteint. On conçoit d'ailleurs aisément que, dans la nature, la compétition intraspécifique et l'action des multiples prédateurs auraient eu raison d'un animal bien avant que 
son organisme ne soit complètement envahi par les larves du Cestode.

Cette observation apporte une preuve de plus au sujet des possibilités des cysticerques de Taenia crassiceps de se reproduire rapidement par bourgeonnement. Il est vraisemblable que les embryons hexacanthes vont normalement se loger dans les tissus souscutanés et que c'est là que débute la reproduction pædogénétique. Par la suite, les larves filles traversent les membranes et envahissent la cavité générale de l'hôte. La compression exercée par la masse des cysticerques sur certains organes entraîne des troubles fonctionnels plus ou moins graves.

Dans les conclusions de son travail, Arvy (1) émettait l'hypothèse que la cysticercose due aux larves de $T$. crassiceps, en stérilisant les mâles atteints, puisse être responsable des diminutions brutales de populations observées chez différents Rongeurs, dont Microtus arvalis. A notre avis, le cas de stérilisation observé par cet auteur est, sinon exceptionnel, du moins rare. Comme nous le signalons plus haut, dans la nature, la pression biotique du milieu doit détruire l'animal atteint bien avant que l'affection n'ait pu entraîner la sénilité précoce. De plus, en Belgique, le nombre d'animaux frappés par cette cysticercose est toujours très faible.

En conclusion, nous ne pensons pas que cette affection parasitaire entre pour une part importante dans le complexe de facteurs responsables des disparitions massives de campagnols qui suivent les maxima de population.

\section{Station d'Entomologie, Gembloux}

\section{BIBLIOGRAPHIE}

1. ARvy (L.), 1950. - Contribution à l'étude histologique de la cysticercose chez Microtus arvalis Pall.. Rev. canad. Biol., 9, pp. 368-381.

2. Rausch (R.), 1952. - Studies on the helminth fauna of Alaska. XI. Helminth parasites of Microtine Rodents. Taxonomic considerations. J. of Parasitology, 38,5 , pp. 415-442. 